\title{
Hérnia de Spiegel: Relato de dois Casos
}

\author{
Herniated Spiegel: Report of two Cases \\ Victor Carlos Saiz Vieira ${ }^{\dagger *}$, Richard Raphael Borges Tavares Vieira ${ }^{\dagger}$, Thais Barbosa Alves ${ }^{\dagger}$, \\ Amanda Guimarães de Souza ${ }^{\dagger}$, Joaquim Ferreira de Paula ${ }^{\dagger}$, Carlos Augusto Marques \\ Batista $^{\dagger+}$
}

Como citar esse artigo. Vieira VCS, Vieira RRBT, Alves TB, de Souza AG, de Paula JF, Batista CAM. Hérnia de Spiegel: Relato de dois Casos Revista de Saúde. 2016 Jul/Dez.; 07 (2): 26-30.

\begin{abstract}
Resumo
As hérnias de Spiegel ocorrem por defeito da parede abdominal através da aponeurose spigeliana, entre a linha semilunar e a borda lateral do músculo reto, logo abaixo da linha arqueada de Douglas e, geralmente, acima dos vasos epigástricos superiores. Este defeito pode se estender desde a nona cartilagem costal até a espinha púbica e recebeu esse nome em homenagem ao anatomista belga Adriaan van den Spiegel, após detalhar as estruturas anatômicas envolvidas nesse tipo de hérnia. O seu pequeno e fibroso colo é responsável por estrangulamento em boa parte dos casos. Estas hérnias são de grande interesse para o cirurgião pelos vários diagnósticos diferenciais que se pode levantar. Geralmente, não apresentam sintomas característicos, podendo nem mesmo haver massa evidente à inspeção ou palpação. $\mathrm{O}$ objetivo deste trabalho é relatar achados compatíveis com as hérnias de Spiegel, defeitos estes muito raros e com poucos relatos na literatura mundial.

Palavras-Chave: Hérnia de Spiegel; Aponeurose Spigeliana; Cinturão da Hérnia Spigeliana
\end{abstract}

\begin{abstract}
The Spiegel hernias occur by defect of the abdominal wall through spigeliana aponeurosis between the lunate line and the lateral border of the rectus muscle just below the arcuate line of Douglas and generally above the upper epigastric vessels. This defect may extend from the ninth costal cartilage to the pubic spine and was named in honor of the Belgian anatomist Adriaan van den Spiegel after detailing the anatomical structures involved in this type of hernia. Its small and fibrous lap is responsible for strangulation in most of cases. These hernias are of great interest to the surgeon by the various differential diagnoses that can lift. Generally they do not have characteristic symptoms and may not even be mass evident inspection or palpation. The objective of this study is to report findings consistent with hernias Spiegel, which are very rare with few reports in the literature.

Keywords: Herniated Spiegel; Aponeurosis Spigeliana; Belt Hernia Spigeliana
\end{abstract}

As hérnias de Spiegel apresentam-se como um defeito da parede abdominal, ocorrendo através da aponeurose spigeliana, entre a linha semilunar e a borda lateral do músculo reto, logo abaixo da linha arqueada de Douglas e, geralmente, acima dos vasos epigástricos superiores, área esta denominada de zona de Spiegel. Este defeito pode se estender desde a nona cartilagem costal até a espinha púbica. Recebeu esse nome em homenagem ao anatomista belga Adriaan van den Spiegel após detalhar as estruturas anatômicas como a linha semilunar, envolvidas nesse tipo de hérnia. ${ }^{1,2}$

Seu pequeno e fibroso colo pode levar ao estrangulamento em até $30 \%$ dos casos e os órgãos que geralmente encarceram-se são: intestino delgado, cólon ou omento. ${ }^{3}$ Além disso, já foram descritos encarceramento do estômago, vesícula biliar, ovário e apêndice. ${ }^{4,5}$ Estas hérnias são de grande interesse para o cirurgião pelos vários diagnósticos diferenciais que se pode levantar e por não apresentarem sintomas característicos, podendo ainda ocorrer de forma intraparietal, sem massas evidentes à inspeção ou palpação. ${ }^{2-5}$

As hérnias de Spiegel caracterizam-se por serem raras e com diagnóstico muitas vezes de exclusão, principalmente quando apresentam somente dor como sintoma. Na grande maioria dos casos, pode conter o intestino delgado, cólon sigmoide, cólon transverso, ceco, apêndice, ou divertículo de Meckel. ${ }^{1-5}$ É considerado um defeito congênito ou adquirido, e quando adquirido, costuma se desenvolver durante a quarta e a sétima década de vida..$^{1,5-7}$

O tratamento é fundamentalmente cirúrgico, tendo em vista o importante risco de obstrução e/ou estrangulamento do seu conteúdo e a abordagem atual

\footnotetext{
Afiliação dos autores: †Faculdade de Medicina, Núcleo Biomédico, Centro de Ensino Superior de Valença, Fundação Dom André Arcoverde, Valença/RJ, Brasil ¥ Hospital Escola Luiz Gioseffi Jannuzzi, Fundação Dom André Arcoverde, Valença/RJ, Brasil

* Endereço para correspondência: Osmar Emidio dos Santos, Mãe: Conceição de Lourdes Dovidio dos Santos, Rua Benedito Duarte Mendes, 172, Centro, Urânia, SP email: diego_urania@hotmail.com
} 
baseia-se na herniorrafia sem ou com a colocação de tela. ${ }^{1,3,5-8}$

Este trabalho objetiva a descrição de dois casos de hérnia de Spiegel abordados nas dependências do Hospital Escola Luiz Gioseffi Jannuzzi, com ênfase em aspectos clínicos e diagnósticos, terapêutica empregada e resultado pós-operatório alcançado.

Os pacientes foram informados sobre todos os aspectos do trabalho, tendo concordado em participar e preenchido Termo de Consentimento Livre e Esclarecido, ficando uma via na posse dos pacientes e outra em posse dos pesquisadores, após o aceite do trabalho pelo Comitê de Ética em Pesquisa.

Procedeu-se então, revisão literária a respeito da ocorrência da patologia em questão, incluindo artigos publicados em periódicos indexados ao PubMed e Scielo e utilizando os descritores hérnia de Spiegel e aponeurose spiegeliana. Foram selecionados 10 artigos publicados entre 1989 e 2015 de interesse para o trabalho e após isso, os dados da literatura foram confrontados com os nossos achados.

\section{Relato de Caso 1}

Paciente do sexo feminino, 77 anos, admitida com quadro de vômitos há 5 dias, dor abdominal há 3 dias, parada de eliminação de gases e fezes e massa palpável em fossa ilíaca esquerda. Portadora de hipertensão arterial sistêmica de longa data, sendo tratado com maleato de enalapril (TEUTO) $25 \mathrm{mg} /$ dia. Ao exame físico apresentava-se afebril, eupnêica e desidratada; abdome flácido e distendido, doloroso difusamente, com peristalse preservada e palpação de massa dolorosa e fixa em fossa ilíaca esquerda. Apresentava leucocitose de $22.600,5$ bastonetes e 66 segmentados; uréia $=52$; creatinina $=1,1 ;$ sódio $=134$ e potássio $=3,5$.

Com as hipóteses diagnósticas de obstrução intestinal e de diverticulite aguda a esclarecer, optouse em realizar tomografia computadorizada, que demonstrou hérnia de Spiegel encarcerada a esquerda, sem evidenciar sinais de necrose intestinal. Foi indicada a cirurgia e a paciente classificada como risco cirúrgico ASA III.

No ato operatório, através de incisão transversa sobre o abaulamento, comprovou-se abaulamento de conteúdo herniário com sinais de estrangulamento (Figura 1) e constituído de jejuno necrosado (Figuras 2). Foi realizado enterectomia parcial com anastomose término-terminal seguida de herniorrafia mediante a aproximação das camadas musculoaponeuróticas sem colocação de tela de polipropileno e drenagem a vácuo do espaço formado no subcutâneo.

O exame histopatológico revelou: Peça 1 - corte de jejuno apresentando marcada congestão e dilatação dos vasos da parede, com áreas de necrose da mucosa. Peça 2 - saco herniário mostrando fibrose, congestão dos vasos, reação inflamatória aguda focal.

A realimentação oral ocorreu no $3^{\circ}$ dia de PO e a alta no $7^{\circ}$ dia. Atualmente, com 5 anos de acompanhamento, não apresentou recidiva.

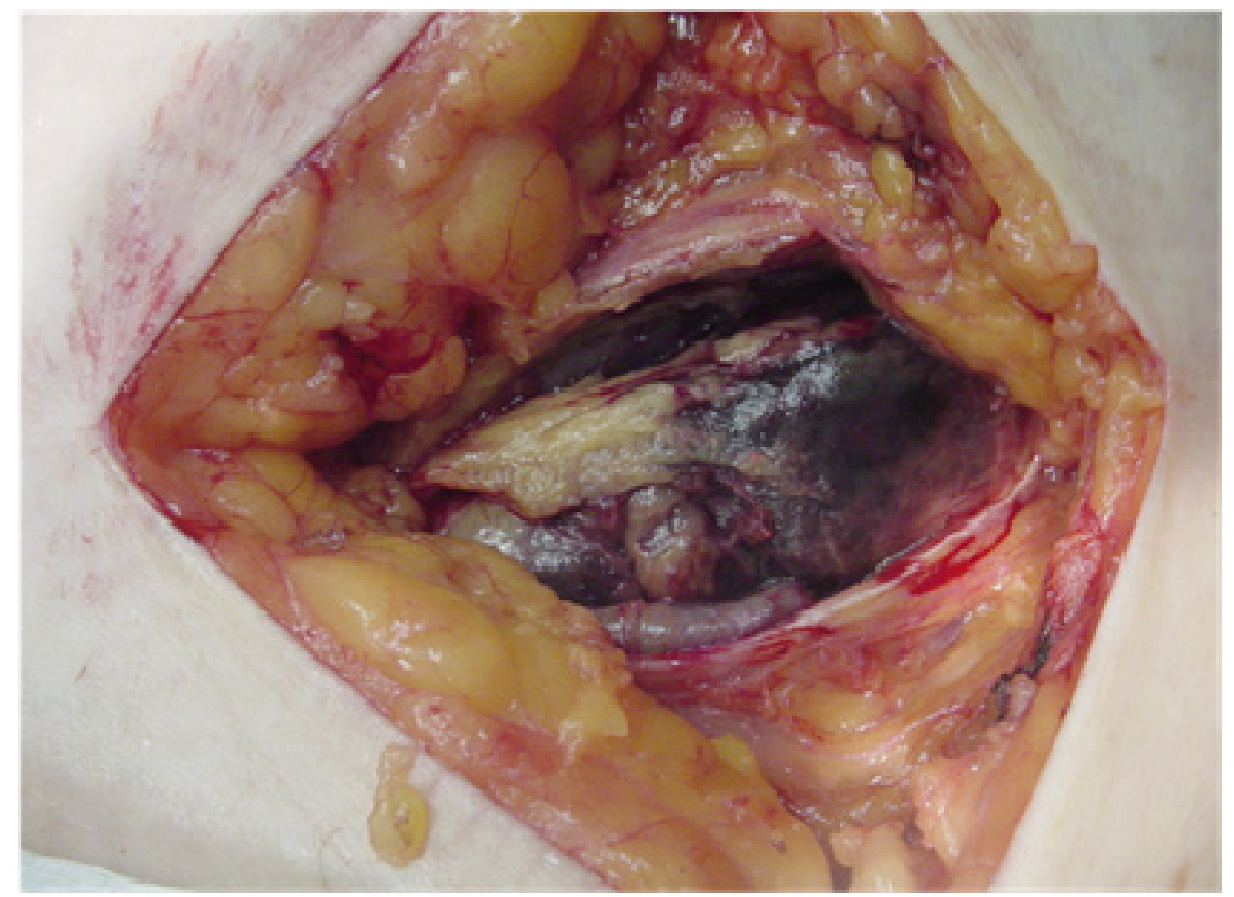

Figura 1. Saco herniário com sinais de estrangulamento. 


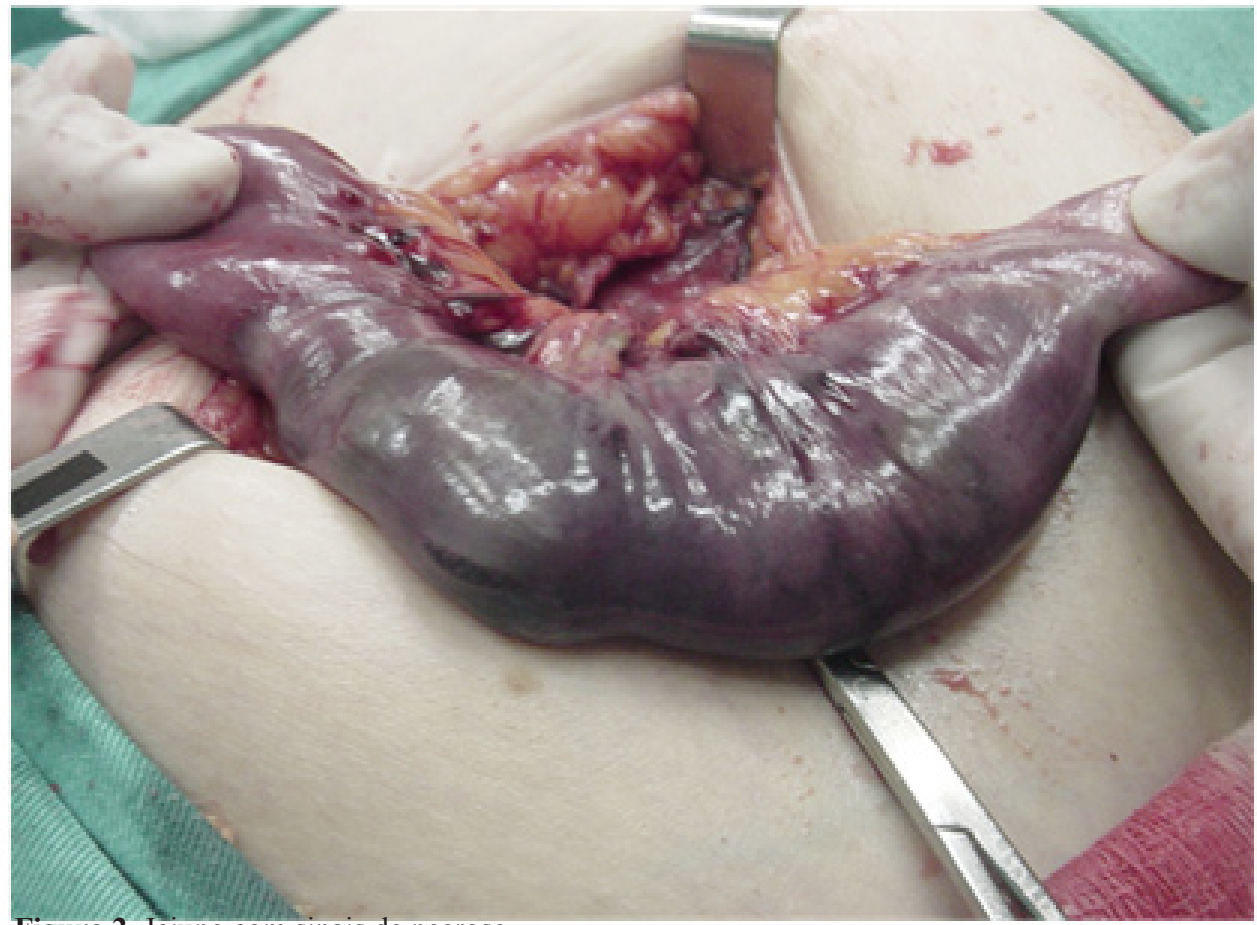

Figura 2. Jejuno com sinais de necrose

\section{Relato de Caso 2}

Paciente do sexo feminino, 79 anos, admitida há 9 dias com história de abaulamento em quadrante inferior direito há cerca de 1 ano, de crescimento rápido, redutível, indolor e sem associação com outras queixas.

História pregressa de histerectomia, portadora de hipertensão arterial sistêmica e dislipidemia, em tratamento com losartana potássica (PRATIDONADUZZI) $50 \mathrm{mg} /$ dia, hidroclorotiazida (TEUTO) $25 \mathrm{mg} /$ dia, besilato de anlodipina (TEUTO) $5 \mathrm{mg} /$ dia e sinvastatina (SANDOZ) $20 \mathrm{mg} / \mathrm{dia}$.

Ao exame físico apresentava-se afebril, eupnêica corada e hidratada; abdome semi-globoso, com peristalse preservada e palpação de massa indolor palpável em quadrante inferior direito.

Para melhor delineamento do defeito musculoaponeurótico, realizou tomografia computadorizada abdominal que confirmou o diagnóstico clínico de hérnia de Spiegel direita, levando a indicação formal da cirurgia. Paciente foi classificada como risco cirúrgico ASA II.

$\mathrm{O}$ ato operatório consistiu de incisão transversa sobre o abaulamento e herniorrafia com dissecção e redução do saco herniário (Figura 3), colocação de tela de polipropileno no espaço pré-peritoneal (Figura 4), reparo dos defeitos musculoaponeuróticos mediante aproximação de suas camadas e drenagem a vácuo do subcutâneo. Evoluiu sem intercorrências, recebendo alta no $2^{\circ}$ dia de pós-operatório.

\section{Discussão}

As hérnias de Spiegel caracterizam-se por serem raras e com diagnóstico muitas vezes de exclusão, devido a sua clínica inespecífica. ${ }^{3}$ Podem ser denominadas de hérnia de Spigel ou Spigelius, hérnia da linha semilunar ou hérnia ventrolateral espontânea do abdome e apresentam protrusão de um saco peritoneal, gordura pré-peritoneal, ou algum órgão por um defeito na região aponeurótica do transverso abdominal, situada entre a linha semilunar e a borda lateral do reto abdominal, na zona de Spiegel. ${ }^{2,5-8}$

Possuem como fatores predisponentes 0 aumento da pressão intra-abdominal, obesidade, pneumobroncopatia crônica, multiparidade, constipação intestinal, ascite, história de traumas ou incisões abdominais prévias e diálise peritoneal crônica. ${ }^{1-6,9} \mathrm{~A}$ maioria dos pacientes apresenta uma clínica inespecífica, podendo ainda a hérniação ocorrer intraparietal, sem massa evidente a inspeção ou palpação; apresentando muitas vezes dor localizada como único sintoma. . $^{5,910}$

$\mathrm{Na}$ maioria dos casos o diagnóstico é clínico, porém o advento da ecografia possibilitou o aumento do número de diagnósticos e possibilitou uma maior segurança no diagnóstico diferencial com apendicite, abscesso apendicular, neoformação da parede abdominal e hematoma da bainha dos retos. ${ }^{3,4}$ A tomografia computadorizada é muito útil é quando essas hérnias se tornam encarceradas ou estranguladas e a cirurgia laparoscópica é pouco utilizada como diagnóstico por requerer invasão da cavidade abdominal com consequente trauma peritoneal e risco de injúria 
Figura 3. Saco herniário dissecado

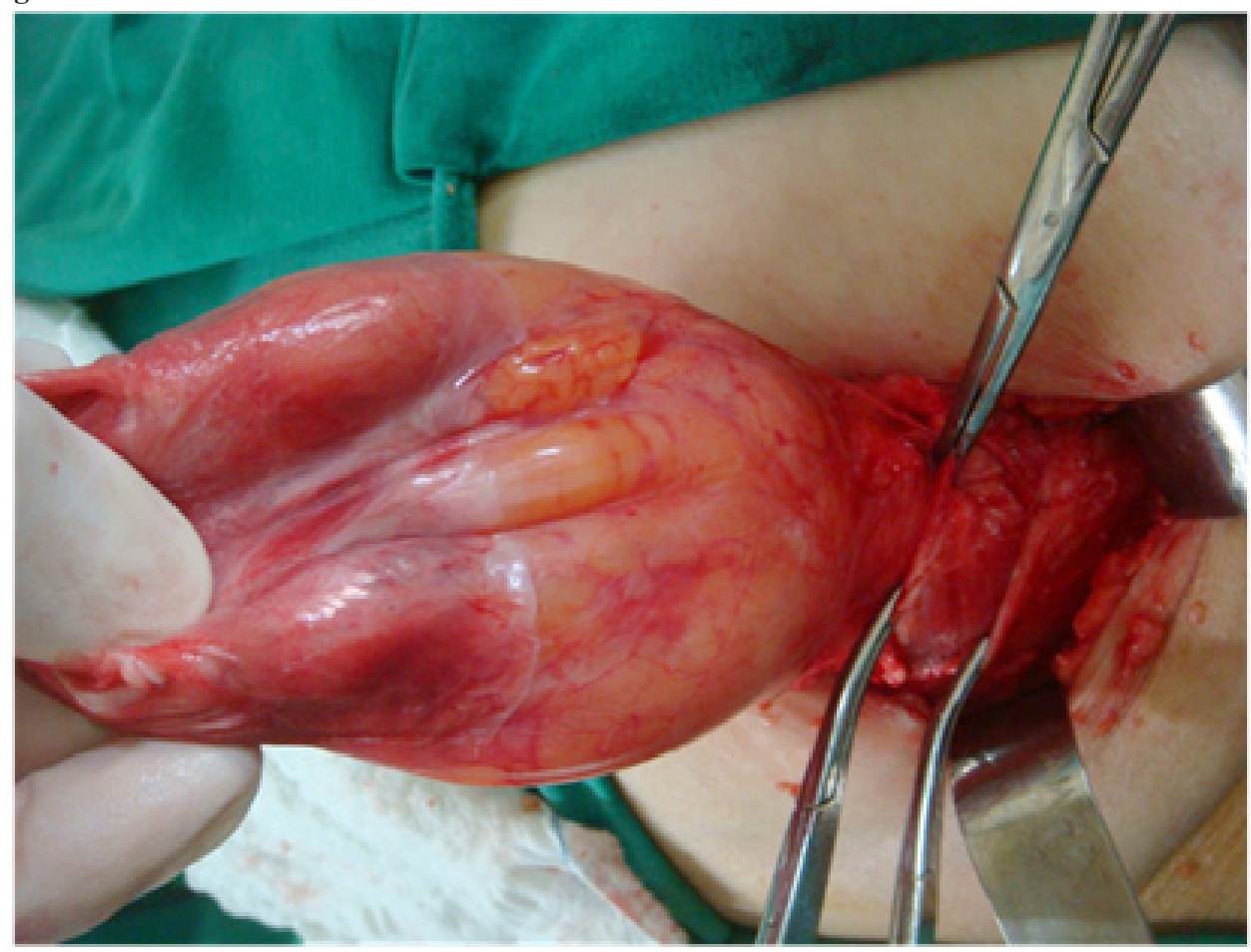

Figura 4. Prótese fixada no espaço pré-peritoneal

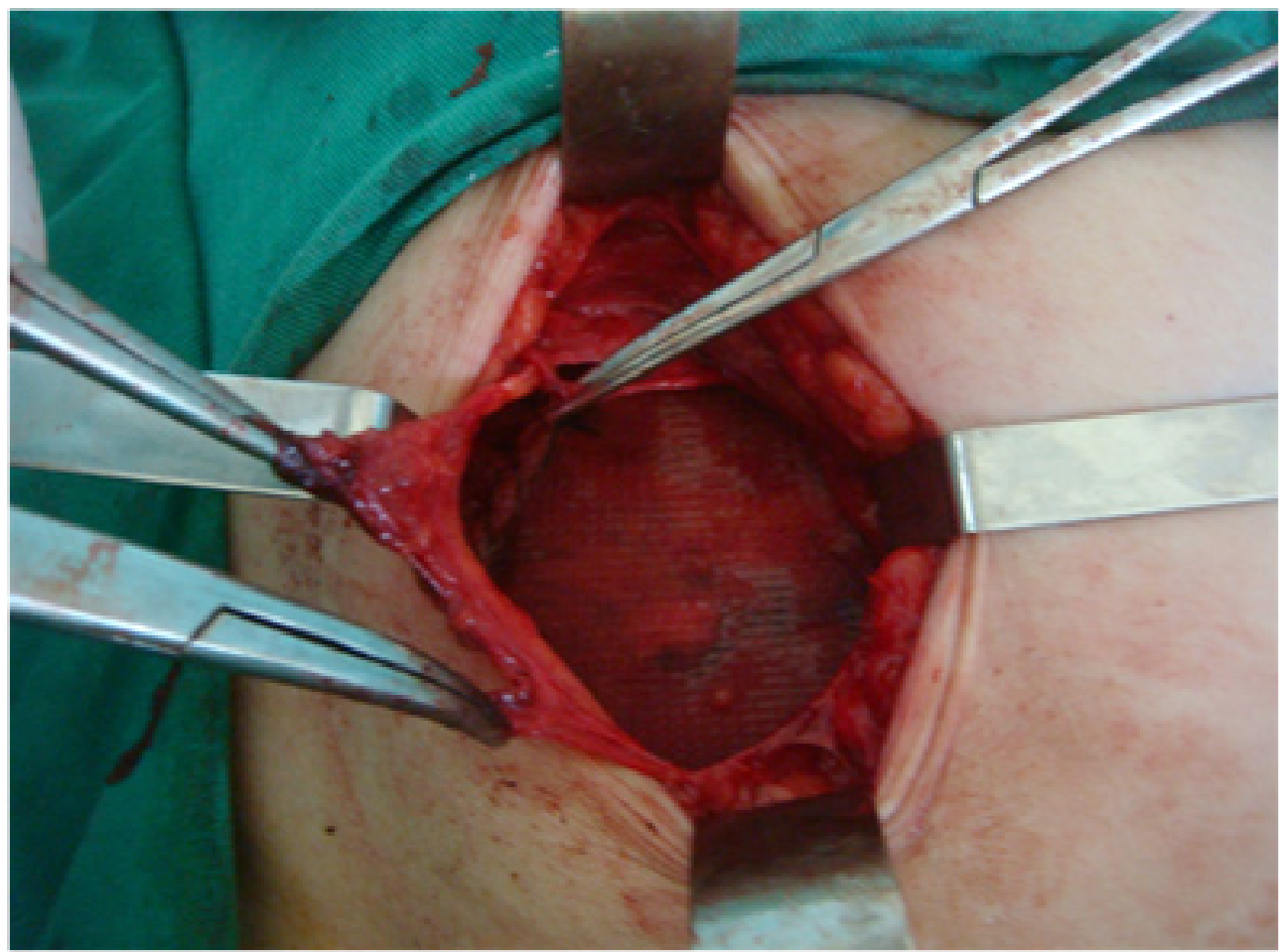

visceral. A ressonância magnética nuclear é o exame que poderá fornecer melhor detalhamento anatômico regional, apesar disso, atualmente a ultrassonografia é a modalidade de escolha para seu diagnóstico. ${ }^{7-10}$

O diagnóstico precoce pode em muitos casos, ser retardado por fatores como ausência de sintomas típicos e a falta da experiência médica com esse tipo de hérnia, sendo ainda mais complicado pelo fato da aponeurose do músculo oblíquo externo encontrar-se intacta, cobrindo o defeito da parede abdominal. ${ }^{6,7}$

A hérnia de Spiegel pode complicar encarcerando ou estrangulando, por isso a importância de se 
diagnosticar e tratar prontamente. A cirurgia é primordial e atualmente as técnicas de hernioplastia pré-peritoneal tension-free tem sido as técnicas de escolha, baseando em uma incisão transversa seguida de reparo primário., ${ }^{1,5-8}$

Nos casos abordados por este serviço, foi utilizada a colocação de tela somente na paciente com risco cirúrgico ASA II - apresentando doença sistêmica sem comprometimento funcional, enquanto a paciente com risco cirúrgico ASA III - doença sistêmica já comprometedora; foi submetida apenas a hernioplastia.

\section{Considerações finais}

Neste trabalho são descritos dois casos de hérnias abdominais. No $1^{\circ}$ caso, foi realizado enterectomia parcial, herniorrafia mediante a aproximação das camadas musculoaponeuróticas sem colocação de tela de polipropileno e drenagem a vácuo do espaço formado no subcutâneo, com realimentação oral ocorrendo no $3^{\circ}$ dia de pós-operatório e a alta no $7^{\circ}$ dia.

No $2^{\circ}$ caso, $o$ ato operatório consistiu de incisão transversa sobre o abaulamento, dissecção e redução do saco herniário, colocação de tela de polipropileno no espaço pré-peritoneal, reparo dos defeitos e drenagem a vácuo do subcutâneo. Evoluiu também sem intercorrências, tendo alta dois dias após a cirurgia.

Ambas foram caracterizadas como spiegelianas por atravessarem a aponeurose spigeliana, logo abaixo da linha arqueada de Douglas. ${ }^{1,2-8}$ Ocorreram em mulheres e com idade maior que 75 anos, corroborando com dados da literatura sobre esta prevalência em faixa etária e gênero. ${ }^{5-7}$

Após o procedimento cirúrgico, houve melhora dos quadros clínicos apresentados e desaparecimento dos abaulamentos, sem qualquer sinal de recidiva anos após a cirurgia.

\section{Referências Bibliográficas}

1. Leme PLS, Soler WV, Terzian HG, Hohne OMP. Hernia de Spiegel: relato de quatro casos. Rev Col Bras Cir 1990 set-out; 17(5):114-8.

2. Leme et al. Estudo Anatômico da Parede Anterior do Abdome em Cadáver e Hérnia de Spiegel. Rev Col Bras Cir 2001 nov-dez; 28(6):414-20.

3. León JS, Acevedo AF, Dellepiane VP. Hérnia de Spiegel* Rev Chil Cir 2011 fev; 63(1):64-8

4 .Flumeri E, Guida M, Torres SL \& Marum M. Hernia de Spiegel: nuestra experiencia en ocho casos. Rev Venez Cir 1989; 42(1):142-4.

5. Parreira JM, Chibata M, Saucedo Jr N, Colatusso RP, Paciornik R. Hérnia de spiegel bilateral: relato de caso e revisão de literatura. ABCD Arq Bras Cir Dig 2007; 20(3):208-11.

6. de Sousa AV, Modena SF, Godinho M, De Paula RA. Hérnia de spiegel. Persp Méd 2006 dez; 17:35-6.

7. Lima Filho A, Cavalcante CC, Marinho DRT, Lopes PVA, Barbuto RC, Viana RFR, Lima TMB. Hernioplastia com tela pré peritoneal no tratamento de hérnia de Spiegel. Rev UNINCOR 2015; 13(2):356-61.
8. Ribeiro EA, Cruz Jr RJ \& Moreira SM. Intestinal obstruction induced by a giant incarcerated Spigelian hernia. Case report and review of the literature. Med J 2005; 123(3):148-50.

9. Guimarães VHM, Andrade CFI, Magalhães GMC \& Guerra SNE. Hérnia de Spiegel após abdominoplastia. Rev Soc Bras Cir Plast 1997 janabr; 12(1): 57-64.

10. Goulart A, Marques H \& Reis M. Hérnia de Spiegel: descrição de caso clínico com análise da literatura. Ver Port Cir 2015; (35): 41-7. 